\title{
Nota Farmacológica: Prevención de la enfermedad cardiovascular con aspirina: resumen de la evidencia y la calidad de la prescripción en Argentina
}

Ricardo Bernztein, Mauricio Monsalvo y Luciana Pozo.

\begin{abstract}
Resumen
Luego de describir la evidencia sobre la eficacia y seguridad que respalda el uso de aspirina en la prevención primaria y secundaría de la enfermedad cardiovascular y algunas otras alternativas de atiagregación -en particular clopidogrel- , así como sus pros, contras e indicaciones de uso; los autores describen con datos propios del sistema REMEDIAR de Argentina, la apropiabilidad de la prescripción de aspirina para la prevención secundaría de la enfermedad coronaria y para la prevención de la enfermedad coronaria en diabéticos.
\end{abstract}

Bernztein R, Monsalvo M y Pozo L. Nota Farmacológica: Prevención de la enfermedad cardiovascular con aspirina: resumen de la evidencia y la calidad de la prescripción en Argentina. Evid. act. práct. ambul. 9(5);153-155. Sep - Oct. 2006.

\section{Introducción}

La enfermedad cardiovascular (ECV) que incluye a la enfermedad coronaria, el infarto de miocardio (IAM) el accidente cerebro vascular (ACV) y la enfermedad vascular periférica, tiene una tasa ajustada de mortalidad de 250,35 por cada 100.000 habitantes $^{1}$ y representa el $30 \%$ de las causas de muerte en la República Argentina. Por año, mueren alrededor de 100.000 personas por enfermedades del sistema circulatorio ${ }^{2}$. La mayor parte de estos episodios ocurren en personas mayores, con factores de riesgo reconocidos como hipertensión, hipercolesterolemia, diabetes o historia de tabaquismo.

El riesgo de sufrir una ECV se denomina riesgo cardíaco global (RCG). La ecuación de Framingham ${ }^{3}$ sirve para predecir el riesgo de sufrir un evento coronario "duro" como infarto o muerte de causa coronaria. Para ello, se necesita conocer la edad y el sexo del paciente, el valor de su colesterol HDL en $\mathrm{mg} \%$, su tensión arterial sistólica en $\mathrm{mmHg}$ con o sin medicación antihipertensiva, el de colesterol total en $\mathrm{mg} \%$ y si fuma.

\section{Evidencia que respalda el uso de aspirina para la prevención} de la enfermedad cardiovascular

Está demostrado el efecto antiagregante plaquetario y antiinflamatorio del ácido acetil salicílico (AAS) en estudios de investigación básica, a través de la acetilación de la $\mathrm{PgH}$ sintetasa e inhibiendo en forma irreversible la cicloxigenasa de las plaquetas y del endotelio vascular 4 .

El tratamiento de antiagregación plaquetaria con AAS a bajas dosis (desde $75 \mathrm{mg}$ diarios) es muy efectivo en la prevención primaria y secundaria de las ECV 5 . En todos estos casos, la recomendación de indicar antiagregación plaquetaria es de por vida.

La aspirina es el tratamiento estándar para la prevención de fenómenos trombóticos en prevención primaria y secundaria. Si bien han aparecido otros fármacos antiplaquetarios (ticlopidina, clopidogrel) todavía no cuentan con evidencia que respalde su eficacia como antiagregantes en prevención primaria ${ }^{6}$.

Un metaanálisis ${ }^{7}$ que incluyó cinco ensayos aleatorizados con más de 55.000 pacientes examinó los efectos del AAS tomado diariamente durante cuatro a siete años, con el objetivo de realizar prevención primaria de ECV en personas mayores de 50 años de edad.

Concluyó que la aspirina reduce el riesgo de IAM en un $28 \%$ y el de otras ECV, un $15 \%$. Por cada 1000 individuos tratados durante ese período se previenen cinco IAM. No se observó incremento en la incidencia de ACV, ni disminución de la mortalidad total ni cardiovascular. Con respecto a la enfermedad vascular global, se redujo significativamente la incidencia de angina de pecho, vasculopatía periférica, accidente isquémico transitorio y necesidad de revascularización. El análisis de subgrupos mostró mayores beneficios en los individuos normotensos. Los individuos asignados a AAS que presentaban una presión arterial sitólica al ingreso menor a $130 \mathrm{mmHg}$ tuvieron mejores resultados que los que presentaban cifras superiores a $140 \mathrm{mmHg}^{8}$.

El aumento de sangrado intracraneal se observó en estudios que habían empleado dosis de 325 a $500 \mathrm{mg}$, no observándose en los que habían empleado 75 a 100mg/día. La posibilidad de efectos adversos (sangrado gastrointestinal o cerebral) y el hecho que no se haya evidenciado una disminución de la mortalidad total, hacen que no pueda recomendarse su empleo sistemático.

Para la prevención primaria de eventos cardiovasculares, la efectividad de la aspirina supera a los riesgos de efectos adversos cuando el riesgo global de enfermedad coronaria a cinco años supera el 3\% (por aplicación de la regla de predicción clínica derivada de la cohorte del Estudio Framingham). Por ejemplo, cada mil individuos tratados con AAS durante cinco años se evitan 4 a 12 IAM y se los expone a un riesgo de 0 a 1 ACV hemorrágico adicional y de 2 a 4 sangrados gastrointestinales. Si el RCG es de $5 \%$ se evitan 6 a 20 IAM, con iguales riesgos de efectos adversos. Por lo tanto, con la evidencia actual deberían recibir bajas dosis de aspirina los individuos con un RCG igual o mayor a $3 \%$ a cinco años ó $6 \%$ a diez años y que no presenten riesgo incrementado de sangrado.

De acuerdo a lo expuesto, todos los sujetos con antecedentes de diabetes y/o eventos coronarios deberían recibir tratamiento antiagregante, esto es prevención secundaria. Son imprescindibles los cambios en el estilo de vida y los tratamientos farmacológicos mencionados para la prevención primaria, sumados a los anticoagulantes, los beta bloqueantes y la rehabilitación cardíaca.

\section{Identificación de los pacientes con indicación preventiva de uso de aspirina}

El mayor riesgo se observa en varones mayores de 45 años, mujeres posmenopáusicas, pacientes con antecedentes familiares de enfermedad coronaria, fumadores de al menos un cigarrillo en el último mes, obesos, hipertensos, dislipémicos y diabéticos. Para el médico general es muy importante identificar estos pacientes. Como dijimos, si se cuenta con información de sus factores de riesgo se puede valorar su riesgo cardiovascular a través de la regla de predicción clínica desarrollada sobre la base de los datos de la cohorte de Framingham. Existen varias calculadoras clínicas disponibles en Internet en forma gratuita, tanto en inglés (http://www.intmed.mcw.edu/clincalc/heartrisk.html) ${ }^{10}$ como en castellano (http://200.47.22.81/riesgo/).

La aplicación de la regla de predicción de Framingham sobre cohortes representativas de muestras poblacionales extranjeras, estima que la mitad de los varones de más de 45 años y de las mujeres a los 50 años de edad tiene un RCG mayor al 3\% a los 5 años ${ }^{11}$.

Si bien no tenemos datos de la precisión de esta regla en nuestra población, consideramos que mientras no contemos con otro instrumento desarrollado localmente, sería válido aplicarla en los Centros de Atención Primaria de la Salud (CAPS) de Argentina con el objetivo de estimar el RCV de nuestros pacientes sobre la base de sus factores de riesgo conocidos como edad, sexo, antecedentes familiares, hábito de fumar, diabetes, hipertensión arterial e hipercolesterolemia, priorizando la valoración del RCG en las personas de 45 a 75 años

La prevención primaria de la ECV con AAS se debe complementar otras estrategias como intervenciones dietéticas, tratamientos para el sobrepeso y la obesidad, ejercicio físico, tratamientos para dejar 
de fumar, medidas tendientes a la evitación del tabaquismo pasivo, moderación de la ingesta de alcohol y sodio, tratamiento con agentes hipolipemiantes (estatinas, fibratos y resinas de intercambio) y tratamiento farmacológico de la hipertensión arterial.

Respecto de la prevención secundaria de la ECV, hay evidencias ${ }^{7}$ que sustentan que en pacientes con alto riesgo para eventos isquémicos, el tratamiento antiplaquetario prolongado con aspirina -a dosis tan bajas como $75 \mathrm{mg} /$ día- reduce significativamente la incidencia de eventos cardiovasculares serios como el IAM no fatal, el ACV isquémico no fatal y la muerte vascular (reducción 25 a 30\% del riesgo relativo) sin aumento de otro tipo de muertes, con una reducción del $16 \%$ en la mortalidad total.

Las diferentes dosis diarias de aspirina utilizadas en los distintos ensayos clínicos producen el mismo efecto, ya sean bajas (menores a 75mg/día) medianas (160 a 325mg/día) o altas (500 a $1500 \mathrm{mg} /$ día). Sin embargo, las dosis altas producen mayor sangrado gastrointestinal y no deben utilizarse. Si bien 75 a 325mg diarios se asocian con un riesgo semejante de sangrado, parece razonable elegir las dosis más bajas de este rango.

No hay evidencia clara de que cualquier otra droga antiplaquetaria sea superior a estas dosis de aspirina. Recientemente, se publicó el estudio CHARISMA (Clopidogrel for High Atherotrombotic Risk and Ischaemic Stabilisation, Management and Avoidance) ${ }^{12}$, un ensayo clínico internacional, multicéntrico, aleatorizado y doble ciego, con un seguimiento medio de 28 meses, diseñado para evaluar el potencial beneficio de la adición de clopidogrel (comparado con placebo) al tratamiento establecido con AAS a dosis bajas (75$162 \mathrm{mg} /$ día) en 15.603 pacientes mayores de 45 años con alto riesgo cardiovascular.

La adición de clopidogrel a la terapia con AAS a dosis bajas no mostró una disminución significativa del riesgo de muerte cardiovascular, infarto o ACV (6,8\% vs. 7,3\%; RR: 0,93; IC95\% 0,83$1,05)$ con respecto a la monoterapia con AAS, salvo en el subgrupo de pacientes con arteriosclerosis sintomática, en quienes hubo mejores resultados cuando se adicionó clopidogrel $(p=0,046)$.

La ausencia de beneficios, el incremento de los riesgos (tasa de hemorragias) y el aumento de los costos, proporciona un argumento claro en contra del uso masivo de la terapia antiagregante combinada en prevención primaria de la enfermedad cardiovascular. Si existen efectos colaterales o una contraindicación fundada, por ejemplo pacientes con alergia conocida a la aspirina, ésta puede reemplazarse por clopidogrel, que parece ser seguro y efectivo, aunque más caro.

EI AAS se contraindica en pacientes con HTA no controlada, pacientes con uso crónico concomitante de anticoagulantes, presencia de úlcera péptica activa, hemofilia y otros desórdenes hemorrágicos, porque aumenta el riesgo de hemorragia severa. Por otro lado, el AAS pierde su efecto preventivo cuando se utiliza un anti inflamatorio no esteroide (AINE) en forma crónica ${ }^{13}$. Recordamos además que el AAS debe administrarse con precauciones en los pacientes asmáticos y durante el embarazo.

\section{Calidad de la prescripción en argentina}

El análisis de uso de AAS reviste gran interés. En principio, la provisión de AAS apunta a la mejoría de un problema de Salud Pública, con un potencial impacto sobre la mortalidad general de la población y sobre la morbilidad cardiovascular: $28 \%$ de disminución de los IAM no fatales en la población de riesgo.

Un estudio realizado por la Federación Argentina de Cardiología en una muestra de 950 pacientes atendidos en dos Centros de Atención Primaria de la Salud (CAPS) de la Ciudad de La Plata ${ }^{14}$, informó que el $48,4 \%$ de la población tiene RCG bajo, 26,2\% leve, $19,6 \%$ moderado y $5,8 \%$, alto.

Si se considera que la indicación de AAS en dosis antiagregante es absoluta en la población con riesgo cardiovascular moderado y alto (probabilidad a cinco años de IAM mayor a $5 \%$ ) más del $25 \%$ de la población mayor de 40 años de edad debería recibirla. A ellos hay que sumar a todas las personas con enfermedad cardiovascular ya establecida y/o diabetes.

Respecto de la calidad en la prescripción de AAS por los prestadores del sistema público de Argentina que trabajan con el plan REMEDIAR, resumimos a continuación los datos de nuestra revisión ${ }^{15}$ efectuada sobre una muestra de más de 13 millones de recetas realizadas entre octubre de 2004 y octubre de 2005 .

Se indicó AAS en el 21\% (10.145 de 48.128) de las recetas que consignaban como diagnóstico único "patologías cardiacas" (isquemia, insuficiencia cardiaca y demás enfermedades cardíacas). Ver figura 1.

Figura 1: frecuencia de prescripción de AAS en pacientes con diagnóstico de cardiopatía.

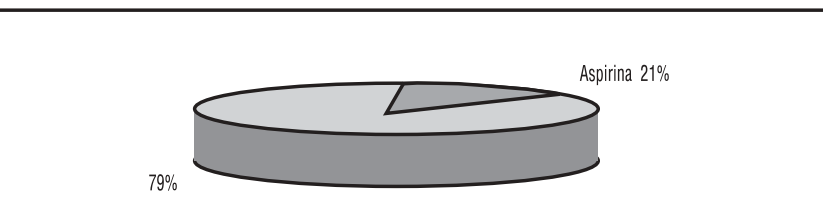

Fuente: Bernztein R, Monsalvo M y Pozo L. Atención Primaria de la Salud, Boletín PROAPS-REMEDIAR 2006; 4 (20):20-9.

En la figura 2 se muestra el análisis de aquellas recetas con diagnóstico único de diabetes mellitus tipo 2, observándose que sólo en el $2 \%$ de las recetas constaba la indicación de AAS (3970 de 196.651).

Figura 2: Frecuencia de prescripción de AAS en pacientes con diagnóstico de diabetes mellitus tipo 2 .

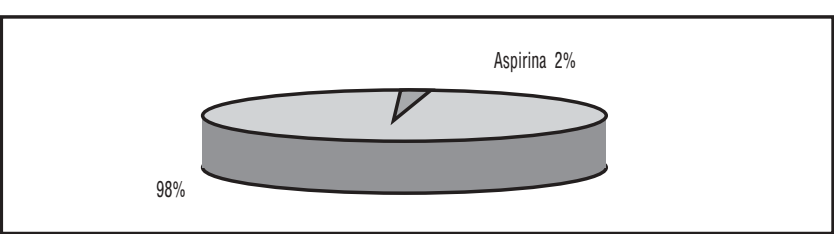

Fuente: Bernztein R, Monsalvo M y Pozo L. Atención Primaria de la Salud, Boletín PROAPS-REMEDIAR 2006; 4 (20):20-9.

Estos datos muestran que, de acuerdo a la evidencia disponible, existe subtratamiento con AAS en el sistema público de atención de Argentina que se provee de medicamentos a través del programa REMEDIAR. La baja tasa de prescripción en esta población de alto riesgo no parece ser explicada por falta de disponibilidad del medicamento, que presentó alto stock en más del $50 \%$ de los CAPS.

\section{Conclusiones}

Lograr que una intervención que ha mostrado eficacia en ensayos controlados sea también efectiva -que evidencie impacto en los indicadores sanitarios- requiere mejorar el perfil prescriptivo, a través de la capacitación de los prescriptores y la difusión de sus beneficios a la comunidad.

Se concluye que el AAS no se prescribió en el PNA público de la República Argentina desde octubre de 2004 a octubre 2005 de acuerdo a la mejor evidencia científica disponible.

Recibido el 25/9/06 y aceptado el 06/10/06. 


\section{Referencias}

1. Situación de Salud en Argentina, 2003. Ministerio de Salud de la Nación, OPS-OMS.

2. Estadisticas Vitales - Dirección de Estadísticas e Información de Salud, Ministerio de Salud y Ambiente de la Nación. En: www.msal.gov.ar

3. Anderson KM, Odell PM, Wilson PW, Kannel WB. Cardiovascular diseaserisk profiles. Am Heart J 1991;121:293-8.

4. Goodman \& Gilman. The Pharmacological Basis of Therapeutics. 10 Ed. Edit Mac Graw, 2001.

5. Doval HD, Tajer C. Evidencias en Cardiología IV. Buenos Aires, Gedic edic. 2005.

6. British Medical Association. British National Formulary: 43rd ed. London: BMA;2002.P469-80.

7. Hayden M, Pignone M, Phillips C, Mulrow C. Aspirin for the primary prevention of cardiovascular events: A summary of the evidence for the U.S. Preventive Services Task Force (USPSTF). Annals of Internal Medicine 2002;136:16172.

8. Meade TW, Brennan PJ on behalf of the MRC General Practice Research Framework. Determination of who may derive the most benefit from aspirin in primary prevention: subgroup results from a randomised controlled trial. BMJ 2000;321:13-17.

9. Wald NJ, Law MR. A strategy to reduce cardiovascular disease by more than $80 \%$. BMJ 2003;326:1419-24.

10. Wilson, PW, et. al. Prediction of Coronary Heart Disease Using Risk Factor Categories. Circulation 1998; 97 (18): $1837-1847$.

11. Caerphilly and Speedwell Collaborative Group. Caerphilly and Speedwell collaborative heart disease studies. J Epidemiol Community Health 1984;38:25962 .

12. Bhatt D et al, the CHARISMA Investigators. Clopidogrel and Aspirin versus Aspirin Alone for the Prevention of Atherothrombotic Events. N Engl J Med 2006; 354:1706-1717.

13. Tobias K et al. Inhibition of Clinical Benefits of Aspirin on First Myocardial Infarction by Nonsteroidal Antiinflammatory Drugs. Circulation, 2003; 108: 119195.

14. Lopez Santi R. et al. Grupo Valoración del Riesgo Cardiovascular Global (VARICG). Riesgo cardiovascular global de una población en un programa de prevención primaria. Rev Fed Arg Cardiol 2003; 32: 358-367.

15. Bernztein R, Monsalvo M y Pozo L. Atención Primaria de la Salud, Boletín PROAPS-REMEDIAR (revista del Programa REMEDIAR) 2006; 4 (20):20-9.

\section{Correo de lectores}

\section{Sr. Director de la Revista Evidencia}

El comentario editorial de la Revista Evidencia de julio-agosto 2006 "Furor diagnosticandis et Furor curandis: Los vientos de la labor médica " me ha impulsado a compartir con Ustedes algunas dudas y reflexiones.

Si bien comprendo la posición de los autores y estoy de acuerdo en general con el concepto, desearía analizar algunos aspectos del editorial:

1) Si esa paciente de 76 años, obesa e hipertensa, que se presenta como caso clínico, hubiera sido tratada con furor curandis 20 ó 30 años atrás, es probable que no hubiera llegado a una insuficiencia renal a punto de ser dializada. Se podrá aducir que en aquella época no existían los fármacos de que disponemos hoy. Pero, frente a entonces a una paciente de similares características que viniera hoy a nuestro consultorio ¿no deberíamos aplicar el furor diagnosticandis y curandis para lograr los objetivos de bajar de peso y cifras tensionales óptimas a la luz de los ensayos clínicos sobre HTA que hemos leído en los últimos años (SHEP, MRFIT, Syst-eur, etc.) así como las guías clínicas (JNC-VII ) que sugieren llegar a tres o cuatro drogas para lograr dicho objetivo y evitar las complicaciones finales de la HTA.

2) Por otra parte, los médicos ¿no nos estaríamos deslizando lentamente hacia el concepto de "inercia clínica " si no aplicáramos ese furor, en el deseo de ayudar al paciente? Se entiende que no es correcto ni un extremo ni el otro, pero ¿cómo lograr el equilibrio? ¿cómo hacer sin llegar al furor y cómo no hacer sin llegar a la inercia? Todo esto en la vorágine solitaria del consultorio.

Algunas respuestas que se me ocurren pueden ser, por ejemplo intentar la categorización del paciente, profundizar la práctica de la medicina basada en la evidencia, estimular las reuniones de discusión clínica con otros profesionales, clínicos y especialistas y probablemente haya más respuestas.

Aprovecho la oportunidad para felicitar a los responsables de la Revista Evidencia, tanto por la prolijidad de la presentación como por sus contenidos.

Agradecido, los saluda atentamente.

\section{Respuesta:}

\section{Estimado Dr Garazzi,}

Muchas gracias por sus comentarios. Siempre es interesante poder intercambiar puntos de vista a través de este medio.

La editorial intenta reflejar más que nada algunas reflexiones y contrastes que fueron surgiendo paulatinamente del intercambio de opiniones entre los autores en relación con el caso citado. El interesante proceso que se desarrolló fue que, partiendo ambos de perspectivas y abordajes diferentes, fuimos coincidiendo en los conceptos sobre los distintos roles que jugábamos nosotros como médicos y el reconocimiento del rol de la paciente como persona autónoma. Esto nos permite ver con algo más de claridad la enorme complejidad de la relación médico - paciente y la absoluta necesidad de contar con información apoyada en bases sólidas, la capacidad de transmitirla a los pacientes y de poder incorporar sus opiniones al proceso de toma de decisiones.

Todos sabemos que el hecho de adoptar un papel dominante o paternalista no garantiza el cumplimiento de metas. Estos logros son, indudablemente, uno de los resultados más estimulantes y gratificantes de la práctica, pero son producto de la interacción sostenida en el tiempo entre nuestras recomendaciones y las decisiones que los pacientes toman en relación a su propio cuidado. El "furor diagnosticandis" y el "furor curandis" son, como ud. bien describe, conductas extremas y como tales, nunca recomendables en la práctica. De la misma manera, la editorial de ninguna manera se inclina hacia el otro extremo de la inercia o la desidia, sino que propugna la dificultosa búsqueda de un equilibrio, para lo cual, nuevamente coincidimos, es necesario desarrollar distintas estrategias, mejorar la comunicación entre los profesionales y, siempre que podamos, incorporar a nuestros pacientes a la mesa del debate.

Carlos Musso

Fernando Rubinstein

\section{Referencias}

1. Musso C, Rubinstein F. Furor Diagnosticandis et Furor Curandis: los vientos de la labor médica. Evid. actual. práct. ambul. 9(4) ;98-99. Jul-Ag. 2006.

2. Prevention of stroke by antihypertensive drug treatment in older persons with isolated systolic hypertension. Final results of the Systolic Hypertension in the Elderly Program (SHEP). SHEP Cooperative Research Group.JAMA. 1991 Jun 26;265(24):3255-64

3. Scott M. Grundy, Richard Pasternak, Philip Greenland, Sidney Smith, Jr, and Valentin Fuster. Assessment of Cardiovascular Risk by Use of Multiple-RiskFactor Assessment Equations : A Statement for Healthcare Professionals From the American Heart Association and the American College of Cardiology. Circulation, Sep 1999; 100: 1481 - 1492.

4. Forette F, Seux ML, Staessen JA, Thijs L, Birkenhager WH, Babarskiene MR, Babeanu S, Bossini A, Gil-Extremera B, Girerd X, Laks T, Lilov E, Moisseyev V, Tuomilehto J, Vanhanen H, Webster J, Yodfat Y, Fagard R. Prevention of dementia in randomised double-blind placebo-controlled Systolic Hypertension in Europe (Syst-Eur) trial.Lancet. 1998 Oct 24;352(9137):1347-51.

5. Expert Panel on Detection, Evaluation, and Treatment of High Blood Cholesterol in Adults. Executive Summary of the Third Report of the National Cholesterol Education Program (NCEP) Expert Panel on Detection, Evaluation, and Treatment of High Blood Cholesterol in Adults (Adult Treatment Panel III). JAMA, May $2001 ; 285: 2486-2497$

6. Ceitlin J. Inercia clínica: otra cara del fracaso terapéutico Evid. Actual. Práct. Ambul.6(4) Julio-Agosto. 2003. 